\section{PM0-053 APPETITE, TASTE AND SMELL CHANGES AFTER WEIGHT LOSS SURGERY}

doi:10.1136/gutjnl-2012-302514b.53

L Graham,* G Murty, D Bowrey. Department of Surgery, University Hospitals of Leicester NHS Trust, Leicester, UK

Introduction It is apparent from day-to-day practice that patients frequently report changes to their appetite, taste and smell after weight loss surgery. There has been surprisingly little written in the literature on this. The aim of the current study was to assess these parameters in a cohort of patients and to explore potential differences between the different types of procedure.

Methods Questionnaires relating to appetite, taste and smell were administered to 264 patients who had undergone weight loss surgery at our institution during the years 2000-2011. Eight of these patients also underwent detailed smell testing using a validated Olfactometer for taste testing for the flavours of sweet, salt, sour and bitter.

Results Responses were received from 133 patients (50\%). Sensory changes in appetite, taste and smell were noted by $95 \%, 68 \%$ and $39 \%$ of patients respectively. Patients who had undergone Roux-en-Y gastric bypass (RYGB) more frequently experienced new aversions to specific foods compared to patients having other types of surgery (RYGB $73 \%$ vs sleeve gastrectomy $40 \%$ vs gastric banding $20 \%$ ), $\mathrm{p}<0.01$. Patients who experienced food aversions experienced a greater level of postoperative weight loss and reduction in BMI, compared to their counterparts without these features. Detailed taste and smell testing did not identify significant changes to smell or taste thresholds after surgery, nor was there a significant correlation between overall taste and smell scores $(p=0.67)$.

Conclusion This study provides preliminary support that patients do experience changes in their appetite, taste and smell following weight loss surgery. These changes need to be investigated further to help support patient education and the informed consent process.

Competing interests None declared.

\section{PM0-054 LAPAROSCOPIC SILASTIC RING LOOP GASTRIC BYPASS (SR-LGBP): A SINGLE CENTRE EXPERIENCE}

doi:10.1136/gutjnl-2012-302514b.54

${ }^{1} \mathrm{M}$ Clarke, ${ }^{*}{ }^{2} \mathrm{~L}$ Pearless, ${ }^{1,2} \mathrm{M}$ Booth. ${ }^{1}$ Department of Surgery, North Shore Hospital, UK; ${ }^{2}$ Surgical Weight Loss Solutions, Waitemata Specialist Centre, Auckland, New Zealand

Introduction Laparoscopic loop gastric bypass (LGBP) may represent a simpler alternative procedure to Roux-en-Y gastric bypass. Placement of a silastic ring (SR) may minimise weight regain. This study reports upto 5-year outcomes following laparoscopic SR-LGBP.

Methods Retrospective analysis of consecutive patients undergoing SR-LGBP between August 2005 and January 2008. A 15-20 ml lesser-curve gastric pouch was created with $32 \mathrm{Fr}$ orogastric bougie, handsewn gastroenterostomy $150-200 \mathrm{~cm}$ distal to the ligament of Treitz and a $6.5-7 \mathrm{~cm}$ silastic ring around the gastric pouch.

Results 156 patients (78\% female, 22\% male) with a mean (range) age of 44 (18-63) years, pre-op weight of 129 (83-197) $\mathrm{kg}$ and BMI of $46(35-64) \mathrm{kg} / \mathrm{m}^{2}$ underwent surgery. $87 \%$ had pre-operative comorbidities and median (range) follow-up was 35 (6-72) months. Mean (SD) \% excess weight loss (EWL) at 6, 12, 24, 36 and 60 months was 74.6 (19.5), 93.4 (21.1), 98.8 (27.6), 93.5 (20.1) and 89 (16.1) respectively. $37 \%$ had complete resolution of comorbidities and $67.3 \%$ required vitamin/mineral supplementation. Overall 90 (57.7\%) patients; $12(7.7 \%)$ early and 78 (50\%) late, reported minor complications, the commonest being reflux (10\%) or vomiting/ dysphagia/food intolerance (16\%). 39 (25\%) patients; 4 (2.6\%) early and $35(22.4 \%)$ late, reported major complications requiring hospi- talisation. Marginal ulcer and anastomotic stenosis were seen in $7.7 \%$ and $9.6 \%$ respectively. $16(10.3 \%)$ patients required reoperation: $6(3.8 \%)$ bile reflux, $3(1.9 \%)$ ring removal, $2(1.3 \%)$ perforation and $5(3.2 \%)$ other. There were no deaths.

Conclusion SR-LGBP achieves excellent EWL with low mortality. While vomiting, food intolerance, reflux, stricture and stoma ulceration were relatively common, only $10 \%$ required reoperation.

Competing interests None declared.

\section{PM0-055 QUALITY OF LIFE FOLLOWING LAPAROSCOPIC BANDED (SILASTIC RING) SLEEVE GASTRECTOMY}

doi:10.1136/gutjnl-2012-302514b.55

${ }^{1} \mathrm{~L}$ Pearless, ${ }^{2} \mathrm{M}$ Clarke, ${ }^{*}{ }^{1,2} \mathrm{M}$ Booth. ' Surgical Weight Loss Solutions, Waitemata Specialist Centre; ${ }^{2}$ Department of Surgery, North Shore Hospital, Auckland, New Zealand

Introduction Placement of a silastic ring around a sleeve gastrectomy (SG) may minimise long-term dilatation and weight regain. This study assessed medium term quality of life outcomes

Methods A questionnaire was sent to 46 patients that underwent surgery between November 2006 and February 2010. During the procedure the stomach was divided $3 \mathrm{~cm}$ proximal to the pylorus Orogastric bougie diameter was 36 French (November 2006-June 2008) or 32 Fr (thereafter). A $6.5-7 \mathrm{~cm}$ diameter silastic ring was placed around the mid-portion of the SG.

Results Responses were received from 29 (63\%) patients (25 female, 4 male) with a mean (range) age of 49 (33-65) years and mean preop BMI of $37.5 \mathrm{~kg} / \mathrm{m}^{2}$. Mean (SD) weight loss and \% excess weight loss at 3 years was 31.1 (10.8) $\mathrm{kg}$ and 90.6 (28.9)\% respectively. 66\% were satisfied with surgery (median Likert score=9) although $48 \%$ reported weight regain. Physical- $-97 \%$ reported food intolerances: meat (59\%), solids (35\%) and vegetables (17\%). 21 (72\%) patients reported vomiting: daily (14\%), twice weekly (14\%), weekly (29\%) or less frequently (43\%). 66\% had reflux, with a median Visick of 2. Exercise capacity increased in $96 \%$ of patients. Emotional-28\% described depression or anxiety affecting their work or other activities. Social-28\% found physical health /emotional problems following surgery interfered with social activities. Compliance- $59 \%$ had blood tests at least annually, 79\% continued multivitamins and $41 \%$ required vitamin/mineral supplementation. Conclusion Placement of a silastic ring around SG as a primary procedure should be avoided due to a high incidence of post-operative reflux, vomiting and food intolerance.

Competing interests None declared.

\section{PM0-056 LAPAROSCOPIC BANDED (SILASTIC RING) SLEEVE GASTRECTOMY: MEDIUM TERM OUTCOMES}

doi:10.1136/gutjnl-2012-302514b.56

${ }^{1} \mathrm{M}$ Clarke, ${ }^{*}{ }^{2} \mathrm{~L}$ Pearless, ${ }^{1,2} \mathrm{M}$ Booth. ${ }^{1}$ Department of Surgery, North Shore Hospital; ${ }^{2}$ Surgical Weight Loss Solutions, Waitemata Specialist Centre, Auckland, New Zealand

Introduction Placement of a band of human dermis around the sleeve gastrectomy (SG) has previously been described to prevent late dilatation and weight regain. The aim of this study was to report our experience using a silastic ring placed around the SG.

Methods 53 patients (male $15 \%$, female $85 \%$ ) with a mean (range) age of $46(23-65)$ years and mean (SD) preoperative BMI of 37.6 (5.3) $\mathrm{kg} / \mathrm{m}^{2}$ underwent surgery between November 2006 and February 2010. The stomach was divided $3 \mathrm{~cm}$ proximal to the pylorus. Orogastric bougie diameter was 36 French (November 2006-June 2008) or 32 Fr (thereafter). A 6.5-7 cm diameter silastic ring was placed around the mid-portion of the SG. A retrospective analysis was performed. 